\title{
The Cosmic Ray - X-ray Connection: Effects of Nonlinear Shock Acceleration on Photon Production in SNRs
}

\author{
Donald C. Ellison \\ Department of Physics, North Carolina State University, Box 8202, Raleigh NC 27695, U.S.A.; don_ellison@ncsu.edu
}

\begin{abstract}
Cosmic-ray production in young supernova remnant (SNR) shocks is expected to be efficient and strongly nonlinear. In nonlinear, diffusive shock acceleration, compression ratios will be higher and the shocked temperature lower than test-particle, Rankine-Hugoniot relations predict. Furthermore, the heating of the gas to X-ray emitting temperatures is strongly coupled to the acceleration of cosmic-ray electrons and ions, thus nonlinear processes which modify the shock, influence the emission over the entire band from radio to gamma-rays and may have a strong impact on X-ray line models. Here we apply an algebraic model of nonlinear acceleration, combined with SNR evolution, to model the radio and X-ray continuum of Kepler's SNR.

Proceedings of the ACE-2000 Symposium on The Acceleration and Transport of Energetic Particles Observed in the Heliosphere, January 5 - 8, 2000, Indian Wells, CA
\end{abstract}

\section{INTRODUCTION}

More than twenty years of spacecraft observations in the heliosphere have proven that collisionless shocks can accelerate particles with high efficiency, i.e., $10-50 \%$ of the ram energy can go into superthermal particles (e.g., Eichler [17]; Gosling et al. [21]; Ellison et al. [19]; Terasawa et al. [30]). Energetic particles exist throughout the universe and shocks are commonly associated with them, confirming that shock acceleration is important beyond the heliosphere as well. In fact, shocks in supernova remnants (SNRs) are believed to be the main source of Galactic cosmic rays, and these shocks are expected to be much stronger than those in the heliosphere and can only be more efficient and nonlinear.

The conjecture that collisionless shocks are efficient accelerators is strengthened by results from plasma simulations, which show efficient shock acceleration consistent with spacecraft observations (e.g., Scholer, Trattner, \& Kucharek [29]; Giacalone et al. [20]), and other indirect evidence comes from radio emission from SNRs (see Reynolds \& Ellison [27]) and equipartition arguments in AGNs and $\gamma$-ray bursts (see Blandford \& Eichler [6] for an early review). There is also clear evidence that shocks can produce strong self-generated turbulence. This has long been seen in heliospheric shocks (e.g., Lee [24, 25]; Kennel et al. [23]; Baring et al. [3]) and there is evidence that it occurs at SNRs as well (i.e., Achterberg, Blandford, \& Reynolds [1]).
While the importance of nonlinear (NL) shock acceleration is evident, NL solutions to diffusive shock acceleration are complicated and results are often unwieldy and difficult to use for astrophysical applications. Therefore, we have developed a simple, algebraic model of diffusive shock acceleration, based on more complete studies, which includes the essential nonlinear effects (Berezhko \& Ellison [4]; Ellison, Berezhko, \& Baring [18]). This technique is computationally fast and easy-to-use, yet includes (i) the modification of particle spectra when the backpressure from energetic ions smooths the shock structure, and (ii) the influences on the shock dynamics when the magnetic turbulence is strongly amplified by wave-particle interactions.

The complications of NL shock acceleration and the many parameters required to characterize it are offset somewhat by the fact that the entire particle distribution function, from thermal to the highest energies, is interconnected and must be accounted for self-consistently with a nonthermal tail connecting the quasi-thermal population to the energetic one. Because energy is conserved, a change in the production efficiency of the highest energy particles must impact the thermal properties of the shock heated gas and vice versa. If more energy goes into relativistic particles, less is available to heat the gas. In contrast, the power laws assumed by test-particle models have no connection with the thermal gas, energy conservation does not constrain the normalization of the power law, and the spectral index can be changed with no feedback on the thermal plasma. Furthermore, there is a direct 
linkage between protons and electrons (which produce most of the photon emission associated with shocked gas) in nonlinear models, so the entire emission from radio to gamma-rays, plus cosmic-ray observations, can, in principle, be used to constrain the models.

Here we describe some of the nonlinear features expected to occur in young SNRs and investigate some implications of efficient cosmic-ray production on the broad-band continuum from Kepler's SNR. We refer to Berezhko \& Ellison [4] and Ellison, Berezhko, \& Baring [18] for details of the NL shock model and its application to particle and photon production in SNRs. Work on the NL X-ray line emission from Kepler is in progress, i.e., Decourchelle, Ellison, \& Ballet [12]. Previous testparticle calculations of the X-ray emission from Kepler have been reported by Borkowski, Sarazin, \& Blondin [7], who used a two-dimensional hydrodynamic simulation, and by Rothenflug et al. [28], who investigated the emission from the reverse shock. The work of Decourchelle \& Ballet [11] is mentioned below.

\section{NONLINEAR SHOCK ACCELERATION}

The nonlinear effects in shock acceleration are of two basic kinds: (i) the self-generation of magnetic turbulence by accelerated particles and (ii) the modification (i.e., smoothing) of the shock structure by the backpressure of accelerated particles. Briefly, (i) occurs when counterstreaming accelerated particles produce turbulence in the upstream magnetic field which amplifies as it is convected through the shock. This amplified turbulence results in stronger scattering of the particles, and hence to more acceleration, quickly leading to saturated turbulence levels near $\delta B / B \sim 1$ in strong shocks. The wave-particle interactions produce heating in the shock precursor which may be observable. Effect (ii) results in the overall compression ratio, $r_{\text {tot }}$, being an ever increasing function of Mach number (as if the effective ratio of specific heats $\gamma_{\text {eff }} \rightarrow 1$ and analogous to radiative shocks), i.e.,

$$
r_{\mathrm{tot}} \simeq 1.3 M_{\mathrm{S} 0}^{3 / 4} \quad \text { if } \quad M_{S 0}^{2}>M_{A 0},
$$

or by

$$
r_{\mathrm{tot}} \simeq 1.5 M_{A 0}^{3 / 8}
$$

in the opposite case $\left(M_{\mathrm{S} 0}\right.$ is the sonic and $M_{\mathrm{A} 0}$ is the Alfvén Mach number). Simultaneously, shock smoothing causes the viscous subshock to be weak $\left(r_{\text {sub }} \ll r_{\text {tot }}\right)$ and the temperature of the shocked gas to drop below the test-particle value.

There are many parameters associated with NL shock acceleration (see Ellison, Berezhko, \& Baring [18] for a listing), but the most important ones that determine the solution are the Mach numbers (i.e., the shock speed, pre-shock density, and magnetic field) and the injection

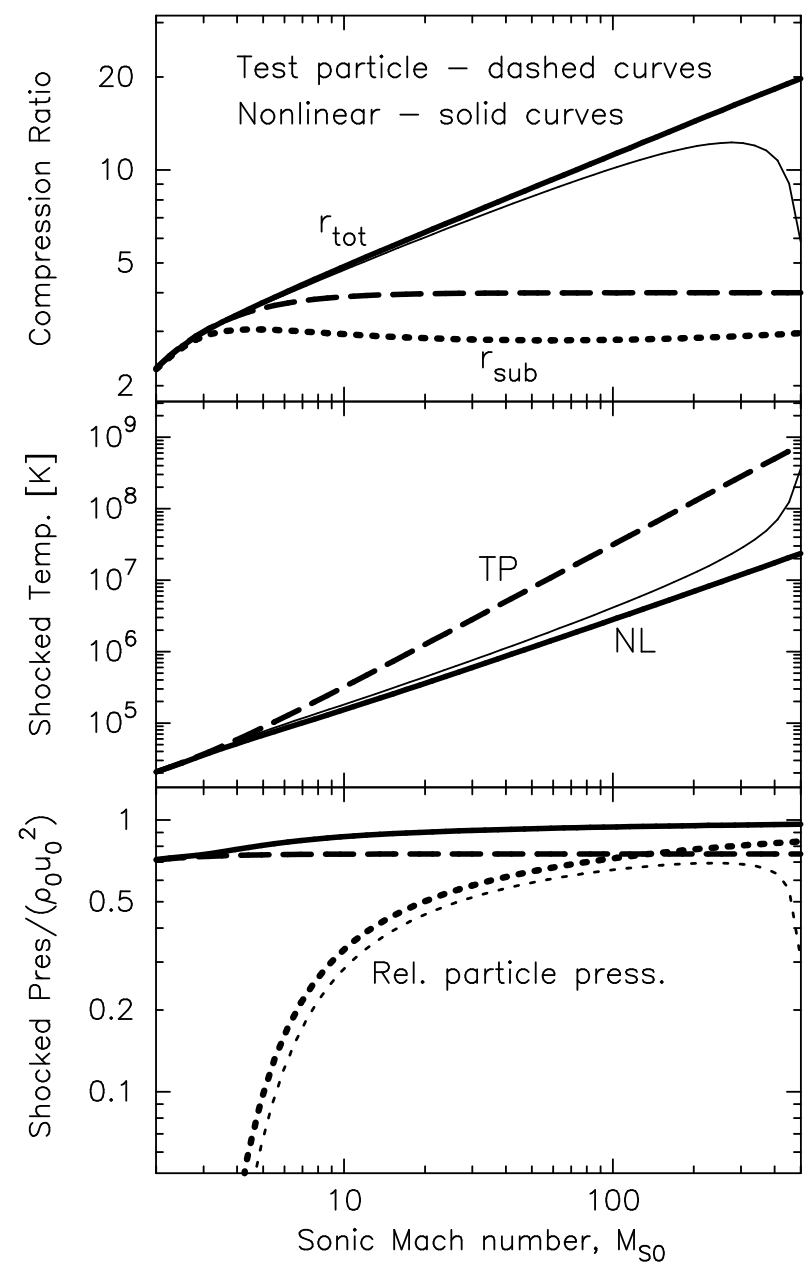

FIGURE 1. Comparison of nonlinear and test-particle shock results as a function of upstream sonic Mach number. The top two panels show that, at $M_{\mathrm{S} 0}>100$, the downstream density can be $>5$ times larger and the shocked temperature $>10$ times smaller if the acceleration is NL. The bottom panel shows that for $M_{\mathrm{S} 0}>100$ and $\eta_{\text {inj,p }}=10^{-3}$, more than $70 \%$ of the pressure in the shocked gas ends up in relativistic particles in NL shock acceleration. These results depend on parameters other than $M_{\mathrm{S} 0}$, and in particular are a strong function of $\eta_{\text {inj,p }}$, particularly at high $M_{\mathrm{S} 0}$. The heavy-weight curves are for $\eta_{\text {inj,p }}=10^{-3}$ and the light-weight curves are for $\eta_{\text {inj,p }}=10^{-4}$.

efficiency, $\eta_{\text {inj,p }}$ (i.e., the fraction of total protons which end up with superthermal energies). As described in Berezhko \& Ellison ([4]), we use Alfvén heating in the precursor which reduces the efficiency compared to adiabatic heating. Significantly, parameters typical of young SNRs should result in NL acceleration.

Figure 1 shows a comparison between test-particle (dashed lines) and nonlinear results as a function of sonic Mach number, $M_{\mathrm{S} 0}$. As illustrated in the top panel, the overall compression ratio, $r_{\text {tot }}$, is generally $>4$ and can 
increase without limit in the NL case for $\eta_{\text {inj,p }}=10^{-3}$. Simultaneously, the subshock compression ratio, $r_{\text {sub }}$, is less than 4 (typically $r_{\text {sub }} \sim 3$ ). The overall compression determines the shocked density, while the subshock is mainly responsible for heating the gas. Thus, the shocked gas will be cooler (middle panel) and denser if the acceleration is NL compared to a test-particle (TP) case. This is an important consideration for X-ray line models. The particle spectrum at the highest energies is determined by $r_{\text {tot }}$, and thus will be flatter than in a TP shock. However, the spectral shape at lower energies depends on $r_{\text {sub }}$, indicating that the particle spectrum will go from being steeper than the TP prediction at low energies to flatter at the highest energies, this is the so-called concave signature of NL shock acceleration. The bottom panel shows that a large fraction of the shocked pressure can end up in relativistic particles (dotted line) for large $M_{\mathrm{S} 0}$. These results depend on the injection efficiency assumed and the turnover in $r_{\text {tot }}$ at large $M_{\mathrm{S} 0}$ seen in the $\eta_{\text {inj.p }}=10^{-4}$ example is a transition to a strong, unmodified shock (see Berezhko \& Ellison [4] for a full discussion).

In Figure 2 we show the phase space distribution functions, $f(p)$, for electrons (dashed line), protons (solid line), and helium (dotted line). The parameters used for this forward shock are those discussed below for Kepler's SNR and given in Table 1. These particles produce photons from synchrotron, bremsstrahlung, inverseCompton, and pion-decay processes (see Baring et al. [2] for details) and these spectra are shown in Figure 3 for both the forward and reverse shocks. As discussed below, we have chosen parameters to cause the reverse shock to contribute substantially to the total X-ray emission, but this choice impacts the emission over the entire range from radio to gamma-rays. While the radio synchrotron comes primarily from the reverse shock, the forward shock dominates emission at $\mathrm{TeV}$ energies with a pion-decay component. Any changes in parameters to accommodate the X-ray observations must be consistent with observational constraints in all other bands.

\section{APPLICATION TO KEPLER'S SNR}

We now apply our model to the radio and X-ray emission seen in Kepler's SNR. The X-ray emission shows the presence of lines and these have been interpreted, in models without acceleration (see Decourchelle \& Petre [13]; Decourchelle et al.. [14]), as coming from the ejecta material heated by the reverse shock. However, these previous models also required the presence of a power law photon continuum, presumably coming from synchrotron emitting TeV electrons (e.g., Reynolds [26]).

Here, we use the evolutionary model of Truelove \& McKee [31] to obtain the forward and reverse shock parameters at the $\sim 395 \mathrm{yr}$ age of Kepler's SNR and, us-

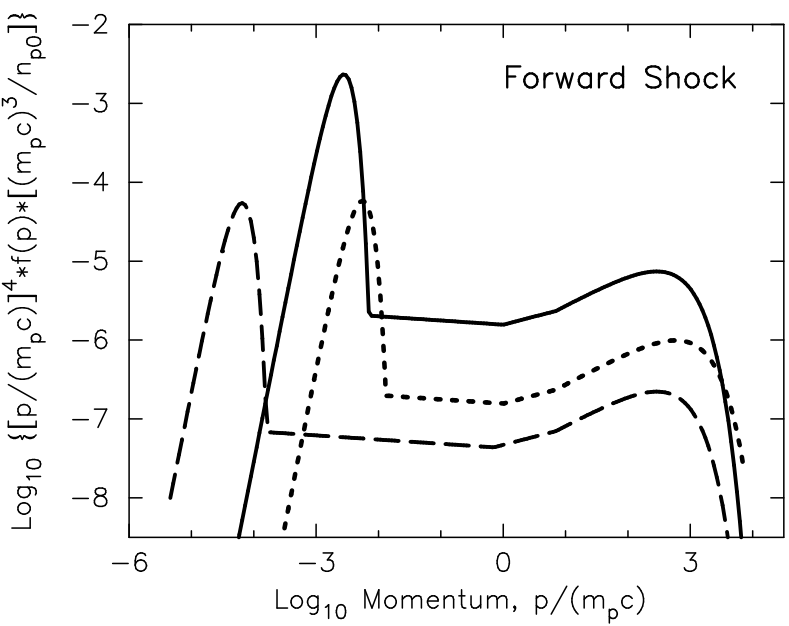

FIGURE 2. Downstream phase space distribution functions, $f$, versus momentum, $p$. We have multiplied $f(p)$ by $\left[p /\left(m_{\mathrm{p}} c\right)\right]^{4}$ to flatten the spectra, and by $\left[\left(m_{\mathrm{p}} c\right)^{3} / n_{p 0}\right]$ to make them dimensionless ( $n_{p 0}$ is the upstream proton number density). The solid line shows protons, the dotted line shows helium, and the dashed line shows electrons.

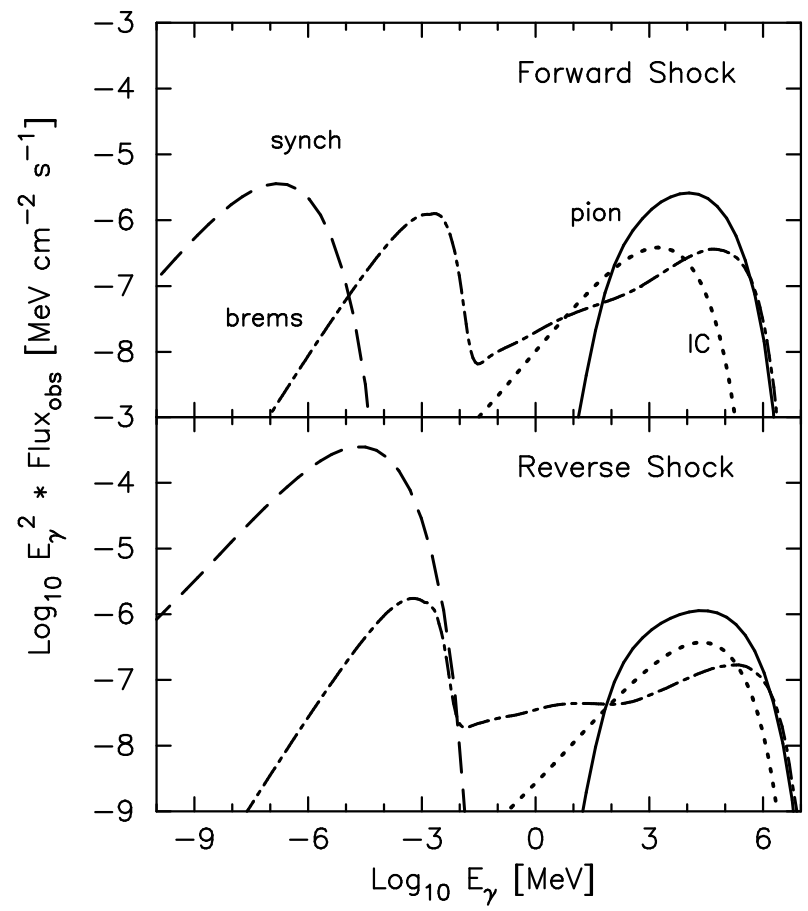

FIGURE 3. Photon spectra from the forward (FS) and reverse shocks (RS) obtained with parameters used to generate the spectra shown in Figure 2. Notice that emission in the X-ray band (1-100 keV) is totally bremsstrahlung for the FS but has a synchrotron component in the RS. Emission at gamma-ray energies is dominated by the FS, while the radio comes totally from the RS. 
ing these shock parameters, calculate the NL particle and then photon spectra. Figure 4 shows the synchrotron and bremsstrahlung emission from Figure 3 and indicates that we are able to obtain a good fit to the data within the constraint that the continuum emission from the reverse shock contribute substantially to the X-ray emission (our model only produces continuum which lies below any Xray emission lines). Since the forward and reverse shocks have very different emission profiles (Figure 3), the ability to resolve the individual shocks in radio and X-rays will be exceptionally important for constraining the models.

We note that the relation between the line and continuum emission is not straightforward and requires a detailed calculation as is currently in progress, i.e., Decourchelle, Ellison, \& Ballet [12]. This is particularly the case in young SNRs because, while the lines and continuum both depend on the electronic temperature in the shocked gas, the lines also depend strongly on the ejecta composition and on the ionization state. The ionization state can be strongly influenced by non-equilibrium effects and by the history of the shocked plasma. What can be said, however, is that the presence of lines restricts the synchrotron intensity to some level comparable to (with a factor of 10 say) or below the bremsstrahlung continuum.

As mentioned above, NL shocks are extremely complicated with many (often poorly defined) parameters and our purpose in this preliminary work is only to give some indication of the issues involved when NL effects are considered. The basic point is that, if nonlinear cosmic-ray production occurs, the $\mathrm{X}$-ray modeling cannot be done without considering emission in other bands, particularly radio and gamma-rays (if available). For Kepler we know that strong lines are present, indicating that the thermal gas behind the reverse shock is contributing substantially to the emission. This strongly constrains the range of acceptable parameters. Previous TP models have also indicated that a continuum component is likely to be present. If so, this is likely to be synchrotron from $\mathrm{TeV}$ electrons and must be consistent with the radio observations.

The parameters used to generate Figure 4 are given in Table 1. All terms in this Table are explained in Ellison, Berezhko, \& Baring ([18]) but some additional explanation is required since we include here, for the first time, a calculation of acceleration at the reverse shock. (i) The unshocked proton number density, $n_{p 0}$, is arbitrarily chosen for the FS and determined for the RS with eqs. (20), (28), and (30) in Truelove \& McKee [31]. (ii) The unshocked magnetic field, $B_{0}$, is arbitrarily chosen and the shocked field (which produces the synchrotron emission) is taken to be $B_{2}=r_{\text {tot }} B_{0}$. We allow for an independent $B_{0}$ for the reverse shock and, in fact, the only way the RS can contribute substantially to the X-ray emission is for the RS $B_{0}$ to be much larger ( 25 vs. $1.5 \mu \mathrm{G}$ ) than

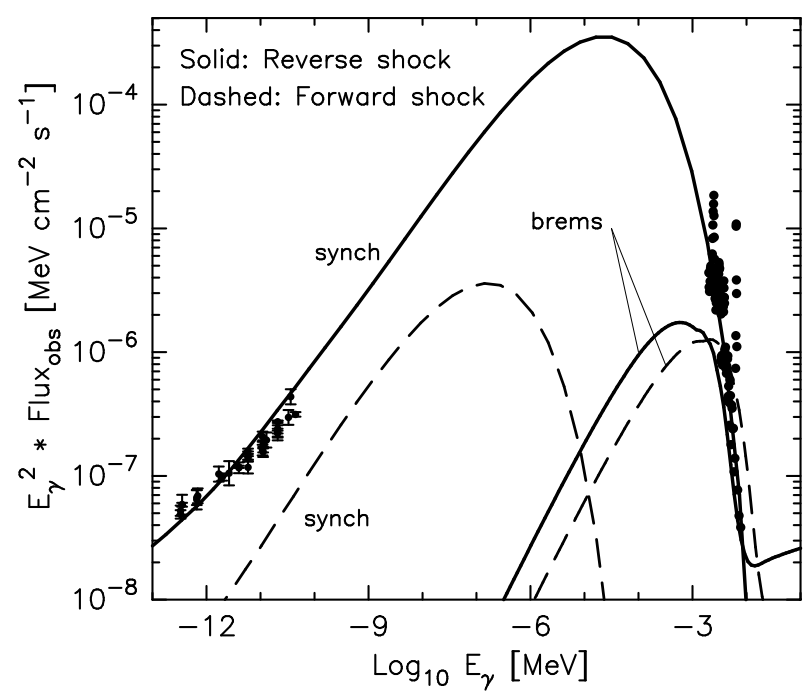

FIGURE 4. Radio and X-ray data for Kepler's SNR compared to the emission from the forward and reverse shocks. The radio data is from Reynolds \& Ellison [27] and X-ray data is adapted from Decourchelle \& Petre [13] (Decourchelle, private communication). We use a standard $v F_{v}$ representation and have suppressed the error bars on the X-ray points.

the FS $B_{0}$. (iii) The supernova energy, $E_{\mathrm{sn}}$, and ejecta mass, $M_{\mathrm{ej}}$, are standard values. (iv) We assume the same unshocked proton temperature, $T_{p 0}=10^{4} \mathrm{~K}$, for the FS and RS. The solutions are relatively insensitive to this parameter. (v) The shocked electron to proton temperature ratio, $T_{\mathrm{e} 2} / T_{\mathrm{p} 2}$, is a free parameter in the NL shock model and is set to 1 for both shocks. (vi) The injection efficiency, $\eta_{\text {inj,p }}$, is an important model parameter determining the overall acceleration efficiency. Values much larger than $\eta_{\text {inj,p }}=2 \times 10^{-4}$ tend to produce strong nonthermal tails on the bremsstrahlung emission which may be inconsistent with the observations. Values much smaller than $2 \times 10^{-4}$ yield test-particle solutions with typical temperatures higher than allowed by the X-ray observations. We note that adjustments in $\eta_{\text {inj,p }}$ produce extremely large modifications in the $\mathrm{TeV}$ results and can be highly constrained with gamma-ray observations. (vii) The electron to proton ratio at relativistic energies, $(e / p)_{\text {rel }}$, is an arbitrary parameter in the simple model but is expected to be between 0.01 and 0.05 if $(e / p)_{\text {rel }}$ in galactic cosmic rays is typical of that produced by the strong shocks in young SNRs. This factor is very important for determining the pion-decay contribution to $\mathrm{TeV}$ gamma-rays. Furthermore, lowering $(e / p)_{\text {rel }}$ lowers the overall emission in the radio to $\mathrm{X}$-ray band but increases the relative contribution of thermal to synchrotron photons in the Xray band. A firm determination of the synchrotron contribution to X-rays, combined with a gamma-ray detection, will help determine $(e / p)_{\text {rel }}$, one of the most important 
unknown parameters in shock acceleration. (viii) The maximum energy cosmic rays obtain in the NL model depends on the scattering mean free path, $\lambda$, which is assumed to be,

$$
\lambda=\eta_{\operatorname{mfp}} r_{\mathrm{g}, \max }\left(r_{\mathrm{g}} / r_{\mathrm{g}, \max }\right)^{\alpha},
$$

where $\eta_{\mathrm{mfp}}$ is taken to be independent of particle momentum (Baring et al. [2], use the notation $\eta$ ), $r_{\mathrm{g}}=p /(q B)$ is the gyroradius in SI units, $r_{\mathrm{g}, \max }$ is the gyroradius at the maximum momentum, $p_{\max }$, and $\alpha$ is a constant parameter $\left(\alpha=1=\eta_{\mathrm{mfp}}\right.$ is roughly the Bohm limit). Here we only consider $\alpha=1$. A fairly large value of $\eta_{\mathrm{mfp}}=15$ (giving a maximum cosmic-ray energy well below that obtained in the Bohm limit) is required to avoid having the X-ray emission totally dominated by synchrotron from $\mathrm{TeV}$ electrons. (ix) We assume that the initial density profile in the ejecta has a power law density distribution, $\rho \propto r^{-n}$, with $n=9$, and that the unshocked ISM is uniform.

The output values are also given in Table 1. Of these, $V_{\mathrm{sk}}$ and $R_{\mathrm{sk}}$ are taken directly from the Truelove \& McKee [31]) solution. Once these are obtained, the Mach numbers are determined (given the relevant input parameters), and then the NL shock model determines the compression ratios, temperatures, and particle spectra. Using the particle spectra, the continuum emission is calculated. It is important to notice that the shocks are highly efficient and NL. They yield total compression ratios $>4$, shocked temperatures nearly 10 times lower than the corresponding test-particle shocks, and place the majority of the total energy flux, $\varepsilon_{\text {rel }}$, into relativistic particles (mainly pro-

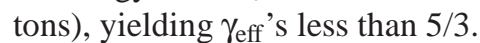

Finally, our model includes a rough estimate of the emission volume

$$
V_{\mathrm{emis}} \approx(4 \pi / 3) R_{\mathrm{sk}}^{3} / r_{\mathrm{tot}}
$$

as described in Ellison, Berezhko, \& Baring [18]. This volume is considerably less than the total remnant volume. If we assume a distance to Kepler of $D_{\mathrm{snr}}=5$ kpc (e.g., Decourchelle \& Ballet [11]), the normalization given by eq. (4), and shown in the Table, matches the observations.

\section{DISCUSSION AND CONCLUSIONS}

$\mathrm{X}$-ray line and continuum emission contains a vast amount of information on supernova (SN) ejecta elemental composition, ISM density and elemental composition, the SN explosion energy, and the mass of ejecta. In addition to heating the plasma, the forward and reverse shocks accelerate some fraction of the shocked material to cosmic-ray energies and this acceleration is believed to be quite efficient, removing energy from the thermal

\begin{tabular}{|c|c|c|}
\hline Input parameters & Forward shock & Reverse shock \\
\hline$n_{p 0}\left[\mathrm{~cm}^{-3}\right]$ & 0.5 & 1.25 \\
\hline$B_{0}[\mu \mathrm{G}]$ & 1.5 & 25 \\
\hline$E_{\mathrm{sn}}\left[10^{51} \mathrm{erg}\right]$ & 1 & - \\
\hline$M_{\mathrm{ej}}\left[M_{\odot}\right]$ & 5 & - \\
\hline$T_{p 0}[\mathrm{~K}]$ & $10^{4}$ & $10^{4}$ \\
\hline$T_{\mathrm{e} 2} / T_{\mathrm{p} 2}$ & 1 & 1 \\
\hline$\eta_{\text {inj.p }}$ & $2 \times 10^{-4}$ & $2 \times 10^{-4}$ \\
\hline$(e / p)_{\mathrm{rel}}$ & 0.03 & 0.03 \\
\hline$\eta_{\mathrm{mfp}}$ & 15 & 15 \\
\hline$n$ & 9 & 9 \\
\hline \multicolumn{3}{|l|}{ Output values } \\
\hline$V_{\mathrm{sk}}\left[\mathrm{km} \mathrm{s}^{-1}\right]$ & 4100 & 1750 \\
\hline$R_{\mathrm{sk}}[\mathrm{pc}]$ & 2.5 & 2.1 \\
\hline$M_{\mathrm{S} 0}$ & 260 & 110 \\
\hline$M_{\mathrm{A} 0}$ & 1040 & 43 \\
\hline$r_{\text {tot }}$ & 11 & 6.4 \\
\hline$r_{\text {sub }}$ & 3.9 & 3.5 \\
\hline$B_{2}[\mu \mathrm{G}]$ & 16 & 160 \\
\hline$E_{\max , \mathrm{p}}[\mathrm{eV}]^{*}$ & $4.1 \times 10^{11}$ & $1.7 \times 10^{12}$ \\
\hline$\eta_{\text {inj,e }}{ }^{\dagger}$ & $5.1 \times 10^{-4}$ & $6.3 \times 10^{-4}$ \\
\hline$T_{\mathrm{p} 2}[\mathrm{~K}]$ & $2.8 \times 10^{7}$ & $1.3 \times 10^{7}$ \\
\hline$T_{\text {tp }}[\mathrm{K}]^{* *}$ & $2.0 \times 10^{8}$ & $9.4 \times 10^{7}$ \\
\hline$\varepsilon_{\mathrm{rel}}$ & 0.79 & 0.54 \\
\hline$\gamma_{\text {eff }}$ & 1.40 & 1.45 \\
\hline \multicolumn{3}{|l|}{ Flux parameters } \\
\hline$D_{\mathrm{snr}}[\mathrm{kpc}]$ & 5 & - \\
\hline$V_{\mathrm{emis}}\left[\mathrm{pc}^{3}\right]$ & 6.0 & 6.4 \\
\hline
\end{tabular}

Table 1. Parameters for Kepler's SNR model

* This is the maximum proton energy, but since synchrotron losses are unimportant here, electrons have the same $E_{\max }$.

$\dagger$ This is the electron injection efficiency.

** This is the temperature the shock gas would have obtained if no acceleration took place.

plasma (e.g., Kang \& Jones [22]; Dorfi \& Böhringer [16]; Berezhko, Ksenofontov, \& Petukhov [5]). Despite the expected efficiency of shock acceleration, virtually all current X-ray line models assume that the shocks that heat the gas do not place a significant fraction of their energy in cosmic rays (exceptions to this are Chevalier [10] and Dorfi [15]). Here, we investigated the broad-band continuum emission expected in Kepler's SNR from efficient shock acceleration, by coupling self-similar hydrodynamics (Chevalier [10]; Truelove \& McKee [31]) with nonlinear diffusive shock acceleration (e.g., Berezhko, Ksenofontov, \& Petukhov [5]). We were able to show that the radio and X-ray continuum can be fit with reasonable parameters in a way that allows the reverse shock to 
contribute substantially to the total X-ray emission. This constraint is required since X-ray line models of Kepler (e.g., Decourchelle \& Petre [13]) require emission from the shock-heated, metal-rich ejecta material.

This preliminary calculation is not fully self-consistent for several reasons. Most importantly, we use self-similar results (i.e., Truelove \& McKee [31]) to model the SNR evolution. These solutions neglect the effects of energetic particle escape from the FS and assume that the ratio of cosmic-ray pressure to total pressure at the shock front is a constant. They also assume that $\gamma_{\text {eff }}=5 / 3$. As indicated in the bottom panel of Figure 1, the total shocked pressure doesn't differ much with or without cosmic-ray production and we have demonstrated (i.e., Decourchelle, Ellison, \& Ballet [12]) that NL shock results do not change dramatically over most of the age of Kepler for typical values of the injection parameter, $\eta_{\text {inj,p }}>10^{-4}$. However for lower $\eta_{\text {inj,p }}$, the nonlinear solutions can have testparticle, unmodified solutions at very high sonic Mach numbers with a rapid transition to the NL solution as the Mach number drops (see Fig. 1 and Berezhko \& Ellison [4] for a detailed discussion). The self-similar solutions we use are still approximate, however, because we have not yet modified them for the change in $\gamma_{\text {eff }}$ that results when a substantial fraction of the shocked pressure is in cosmic rays. We also neglect cosmic-ray diffusion and assume they are spatially coupled to the gas - an excellent approximation for all but the highest energy particles. However, the highest energy electrons produce the X-ray synchrotron photons so there may be differences that are not modeled in the emission volumes and other important parameters between the radio and X-ray bands. Finally, we have not included absorption in our models which is probably not important for the Kepler radio observations, but will be required to model the low energy X-rays. ${ }^{1}$

Besides providing a more self-consistent model of photon production, predictions from NL shock models provide a test of the fundamental assumption that SNRs are the primary source of galactic cosmic-ray ions. If this is so, the acceleration is almost certainly nonlinear since $5-30 \%$ of the total ejecta kinetic energy is required to replenish cosmic rays as they escape from the galaxy. Since shocks put more energy into accelerated ions than electrons, nonlinear effects seen in X-ray emission will be evidence for the efficient shock acceleration of ions as well as electrons. X-ray observations potentially provide in situ information on cosmic-ray ion production, complementing observations of pion-decay $\gamma$-rays in this regard. Any inference of nonthermal tails on electron dis-

\footnotetext{
${ }^{1}$ Note that we have only plotted the X-ray observations above $2 \mathrm{keV}$ in Figure 4 to avoid conflict with the absorbed low energy end of the X-ray distribution.
}

tributions in X-ray observations will provide information on electron injection, the least well understood aspect of shock acceleration. Such in situ information on high Mach number shocks is available nowhere else.

Our modeling of Kepler's SNR suggests that typical source parameters produce large nonlinear effects in the broad-band spectrum and suggest that the test-particle approximations that are almost universally used are inadequate for SNRs as young as this. Besides the differences discussed above, one might expect that the growth rate of the Rayleigh-Taylor instability will be greater in a cosmic-ray modified shock because of the larger spatial gradients of density, pressure, etc. Furthermore, the high compression ratios result in a considerably thinner region between the forward and reverse shocks than predicted in the TP case (Decourchelle, Ellison, \& Ballet [12]). This places the contact discontinuity closer to the shock and may make it easier for the Rayleigh-Taylor "fingers" to distort or overtake the FS, a situation that appears difficult with normal TP parameters (Chevalier, Blondin, \& Emmering [9]; Chevalier \& Blondin [8]). Another important difference concerns electron heating and equilibration. The higher densities in the NL models mean that electron heating is much more efficient than in TP shocks. Our initial calculations [12] suggest that it may be possible to obtain full equipartition between electrons and ions, at least for high values of $\eta_{\text {inj,p }}$, in the shocked ejecta.

\section{ACKNOWLEDGMENTS}

I wish to thank the organizers of the ACE-2000 Symposium for putting on a very useful and enjoyable meeting and for providing support. I'm especially grateful to A. Decourchelle for helpful comments and suggestions and to A. Decourchelle and L. Sauvageot for furnishing the Kepler X-ray data.

\section{REFERENCES}

1. Achterberg, A., Blandford, R.D., Reynolds, S.P. 1994, A.A., 281, 220

2. Baring, M.G., Ellison, D.C., Reynolds, S.P., Grenier, I.A., \& Goret, P., 1999, Ap.J., 513, 311

3. Baring, M.G., Ogilvie, K.W., Ellison, D.C., \& Forsyth, R.J., 1997, Ap.J., 476, 889

4. Berezhko, E.G., Ellison, D.C., 1999, Ap.J., 526, 385

5. Berezhko, E. G., Ksenofontov, L., \& Petukhov, S. I., 1999, Proc. 26th Int. Cosmic Ray Conf. (Salt Lake City), 4, 431.

6. Blandford, R.D., \& Eichler, D., 1987, Phys. Repts., 154, 1

7. Borkowski, K.J., Sarazin, C.L., \& Blondin, J.M., 1994, Ap.J., 429, 710

8. Chevalier, R.A., \& Blondin, J.M., 1995, Ap.J., 444, 312 
9. Chevalier, R.A., Blondin, J.M., \& Emmering, R.T., 1992, Ap.J., 392, 118

10. Chevalier, R.A. 1983, Ap.J., 272, 765

11. Decourchelle, A., \& Ballet, J., 1994, A.A., 287, 206

12. Decourchelle, A., Ellison, D.C., \& Ballet, J., in preparation.

13. Decourchelle, A., \& Petre, R., 1999, Astron. Nachr., 320, 203

14. Decourchelle, A., et al., 2000, in preparation

15. Dorfi, E. A. 1994, Ap.J.Suppl., 90, 841

16. Dorfi, E.A., \& Böhringer, H. 1993, A.A., 273, 251

17. Eichler, D. 1981, Ap.J., 247, 1089

18. Ellison, D.C., Berezhko, E.G., \& Baring, M.G., 2000, Ap. $\mathrm{J}$. , in press- astro-ph/0003188

19. Ellison, D.C., Möbius, E., \& Paschmann, G., 1990, Ap.J., 352,376

20. Giacalone, J., Burgess, D., Schwartz, S.J., Ellison, D.C., \& Bennett, L. 1997, J.G.R., 102, 19,789

21. Gosling, J.T., Asbridge, J.R., Bame, S.J., Feldman, W.C., Zwickl, R.D., Paschmann, G., Sckopke, N., and Hynds, R.J. 1981, J.G.R., 86, 547

22. Kang, H. \& Jones, T. W. 1991, M.N.R.A.S., 249, 439

23. Kennel, C.F., Edmiston, J.P., Scarf, F.L., Coroniti, F.V., Russell, C.T., Smith, E.J., Tsurutani, B.T., Scudder, J.D., Feldman, W.C., Anderson, R.R., Moser, F.S., and Temerin, M., 1984, J.G.R., 89, 5436

24. Lee, M.A., 1982, J.G.R., 87, 5063

25. Lee, M.A., 1983, J.G.R., 88, 6109

26. Reynolds, S.P. 1996, Ap.J.(Letts), 459, L13

27. Reynolds, S.P., \& Ellison, D.C. 1992, Ap.J.(Letts), 399, L75

28. Rothenflug, R., Magne, B., Chieze, J.P., \& Ballet, J., 1994, A.A., 291, 271

29. Scholer, M., Trattner, K.J., \& Kucharek, H. 1992, Ap.J., 395,675

30. Terasawa, T., et al. 1999, Proc. 26th Int. Cosmic Ray Conf. (Salt Lake City), 6, 528.

31. Truelove, J.K., \& McKee, C.F.: 1999, Ap.J.Suppl., 120, 299 